\title{
APPLICATION OF RECIPROCAL INTERVENING VARIABLES FOR STOCHASTIC FINITE ELEMENT ANALYSIS
}

\author{
M. A. Valdebenito ${ }^{1}$, H. A. Jensen ${ }^{1}$, A. A. Labarca ${ }^{1}$ \\ ${ }^{1}$ Universidad Tecnica Federico Santa Maria, Dept. de Obras Civiles, Av. España 1680, Val- \\ paraiso, Chile (\{marcos.valdebenito,hector.jensen\}@usm.cl)
}

\begin{abstract}
This papers investigates the application of first order Taylor expansion considering reciprocal intervening variables for estimating second order statistics of the response of uncertain linear static structural systems. Results presented in this contribution indicate that for estimating second order statistics, the applied expansion is more accurate than approaches based on first and second order Taylor series proposed in the literature while numerical efforts associated with the implementation are similar.
\end{abstract}

Keywords: Stochastic Finite Element Method, Log-normal random field, Second order statistics, Reciprocal intervening variable.

\section{INTRODUCTION}

Within the field of computational mechanics, the Finite Element Method (FEM) is a well established technique for quantifying the response of a structural system [1]. The application of FEM demands an appropriate characterization of the structural behavior and lifetime loadings. However, this characterization can be seldom performed in a precise manner as there are inherent uncertainties associated with structural properties, loadings, etc. Undoubtedly, these uncertainties on the input parameters for the FEM propagate to the structural response. The so-called Stochastic Finite Element Method (SFEM) provides an appropriate tool for quantifying the uncertainty in the structural response [9].

Several different techniques for stochastic finite element analysis have been proposed in the literature [12]. Among these techniques, methods that employ Taylor series expansions (see, e.g $[7,11]$ ) play a fundamental role. In fact, this approach has been termed in the literature as the Perturbation method. The key idea behind Perturbation method is generating an approximate expression for representing the structural response as an explicit function of the uncertain parameters. Methods based on Taylor series expansions are often simple to implement and usually numerically inexpensive, although numerical costs may grow rapidly when the number of uncertain parameters is large and a second order Taylor series is considered. Furthermore, methods based on first-order expansions are usually not very accurate, particularly when the variability in the input parameters is large. 
As mentioned above, the construction of an approximate representation of the structural response plays a pivotal role in the Perturbation method for stochastic finite element analysis. At this point, it should be noted that several methods for structural optimization also deal with the construction of approximate representations of the structural response [5]. Among several different techniques developed for structural optimization, Taylor series expansions have been used widely in a similar way as used for stochastic finite element analysis. However, the application of Taylor series for optimization and stochastic analysis differs in the fact that in the former, the concept of intervening variables is applied extensively [10,5]. The so-called intervening variables allow constructing high quality approximations of the structural response with numerical costs similar to those of a regular Taylor series expansion. Although the concept of intervening variables can be advantageous, its use within the context of SFEM has remained almost unexplored, except by efforts reported in [3], where reciprocal intervening variables are considered for studying trusses whose Young's moduli are modeled by means of uncorrelated random variables. Hence, this contribution studies the application of reciprocal intervening variables and Taylor series expansion for estimating second order statistics of the structural response, focusing on applications involving random field models. In particular, this contribution studies linear, statical structural problems where the Young's modulus is modeled as log-normal random field. The objective is determining whether or not the use of intervening variables and Taylor series expansions can lead to improved estimations of second order statistics when compared to approaches based solely on Taylor series considering no intervening variables, i.e. direct variables.

\section{CHARACTERIZATION OF THE STRUCTURAL RESPONSE}

Assume a structure is modeled using the FEM as linear static, involving $N_{e}$ elements and $N_{d}$ degrees-of-freedom (DOFs). Moreover, its Young's modulus is modeled as an homogeneous log-normal random field and is denoted as $E(\boldsymbol{x})$, where $\boldsymbol{x}$ is a vector describing position. The random field is fully described by its expected value and covariance relation. Applying the midpoint method [2], the random field $E(\boldsymbol{x})$ is represented approximately by its discrete version $\hat{E}(\boldsymbol{x})$. Thus, it can be shown that the Young's modulus at the $p$-th finite element is described by the following expression (see, e.g. [13]).

$$
\hat{E}_{p}=e^{\mu_{p}+\sum_{i=1}^{M} \zeta_{p i} \xi_{i}}, p=1, \ldots, N_{e}
$$

In the above equation, $\mu_{p}$ and $\zeta_{p i}$ are real constants that depend on the expected value and covariance matrix of the discrete random field, $M$ is the number of terms considered when representing the random field using the Karhunen-Loève expansion [8] and $\xi_{i}, i=1, \ldots, M$ are independent standard Gaussian variables.

As the Young's modulus is modeled as a random field depending on the vector of random variables $\boldsymbol{\xi}=\left\langle\xi_{1}, \xi_{2}, \ldots, \xi_{M}\right\rangle^{T}$, the stiffness matrix $\boldsymbol{K}$ (of dimension $N_{d} \times N_{d}$ ) will also depend on this random variable vector, i.e. $\boldsymbol{K}=\boldsymbol{K}(\boldsymbol{\xi})$. Thus, the equilibrium equation is the following.

$$
\boldsymbol{K}(\boldsymbol{\xi}) \boldsymbol{u}(\boldsymbol{\xi})=\boldsymbol{f}
$$

In the eq. (2), $\boldsymbol{u}(\boldsymbol{\xi})$ is the $N_{d} \times 1$ vector of displacements and $\boldsymbol{f}$ is the $N_{d} \times 1$ vector of (equivalent) nodal forces which is assumed to be deterministic for the sake of simplicity. 
Note that the vector of displacements is uncertain as it depends on the random variable vector $\boldsymbol{\xi}$. This is due to the fact the stiffness matrix depends on $\boldsymbol{\xi}$ as well. The uncertainty associated with the displacement vector $\boldsymbol{u}$ is characterized by means of second-order statistics, namely expected value and covariance. For example, the expected value of the displacement of the $n$-th degree of freedom is:

$$
\mathrm{E}\left[u_{n}\right]=\int_{\boldsymbol{\xi} \in \boldsymbol{\Xi}} u_{n}(\boldsymbol{\xi}) f_{\xi}(\boldsymbol{\xi}) d \boldsymbol{\xi}, n=1, \ldots, N_{d}
$$

where $u_{n}$ is the $n$-th component of the displacement vector $\boldsymbol{u}$ and $\mathrm{E}[\cdot]$ denotes expected value. Similarly, the displacement covariance between the $n$-th and $m$-th degree of freedom is:

$$
\operatorname{Cov}\left[u_{n}, u_{m}\right]=\left(\int_{\boldsymbol{\xi} \in \boldsymbol{\Xi}} u_{n}(\boldsymbol{\xi}) u_{m}(\boldsymbol{\xi}) f_{\xi}(\boldsymbol{\xi}) d \boldsymbol{\xi}\right)-\mathrm{E}\left[u_{n}\right] \mathrm{E}\left[u_{m}\right], n, m=1, \ldots, N_{d}
$$

where $\operatorname{Cov}[\cdot, \cdot]$ denotes covariance.

\section{APPROXIMATE REPRESENTATION OF STRUCTURAL RESPONSE CONSID- ERING DIRECT VARIABLES}

Second order statistics of the displacement vector can be estimated based on Taylor expansions. For constructing these expansions, assume the uncertain stiffness matrix $\boldsymbol{K}(\boldsymbol{\xi})$ involved in eq. (2) is expressed in terms of its corresponding Taylor series expansion about $\boldsymbol{\xi}^{0}=\langle 0, \ldots, 0\rangle^{T}$ :

$$
\boldsymbol{K}(\boldsymbol{\xi})=\boldsymbol{K}\left(\boldsymbol{\xi}^{0}\right)+\sum_{i=1}^{M} \boldsymbol{K}_{, i} \xi_{i}+\frac{1}{2} \sum_{i=1}^{M} \sum_{j=1}^{M} \boldsymbol{K}_{, i j} \xi_{i} \xi_{j}+\ldots
$$

where the matrices $\boldsymbol{K}_{, i}$ and $\boldsymbol{K}_{, i j}$ (each of dimension $N_{d} \times N_{d}$ ) are defined as follows.

$$
\begin{gathered}
\boldsymbol{K}_{, i}=\left.\frac{\partial \boldsymbol{K}(\boldsymbol{\xi})}{\partial \xi_{i}}\right|_{\boldsymbol{\xi}=\boldsymbol{\xi}^{0}}, i=1, \ldots, M \\
\boldsymbol{K}_{, i j}=\left.\frac{\partial^{2} \boldsymbol{K}(\boldsymbol{\xi})}{\partial \xi_{i} \partial \xi_{j}}\right|_{\boldsymbol{\xi}=\boldsymbol{\xi}^{0}}, i, j=1, \ldots, M
\end{gathered}
$$

The Taylor series associated with the displacement vector $\boldsymbol{u}(\boldsymbol{\xi})$ about $\boldsymbol{\xi}^{0}=\langle 0, \ldots, 0\rangle^{T}$ is:

$$
\boldsymbol{u}(\boldsymbol{\xi})=\boldsymbol{u}\left(\boldsymbol{\xi}^{0}\right)+\sum_{i=1}^{M} \boldsymbol{u}_{, i} \xi_{i}+\frac{1}{2} \sum_{i=1}^{M} \sum_{j=1}^{M} \boldsymbol{u}_{, i j} \xi_{i} \xi_{j}+\ldots
$$

where the vectors $\boldsymbol{u}\left(\boldsymbol{\xi}^{0}\right), \boldsymbol{u}_{, i}$ and $\boldsymbol{u}_{, i j}$ (each of dimension $N_{d} \times 1$ ) are the nominal displacement, first and second order derivative of the displacement, respectively. These three vectors are defined as (see, e.g. $[9,11])$ :

$$
\begin{aligned}
\boldsymbol{u}\left(\boldsymbol{\xi}^{0}\right) & =\boldsymbol{K}\left(\boldsymbol{\xi}^{0}\right)^{-1} \boldsymbol{f} \\
\boldsymbol{u}_{, i} & =-\boldsymbol{K}\left(\boldsymbol{\xi}^{0}\right)^{-1} \boldsymbol{K}_{, i} \boldsymbol{u}\left(\boldsymbol{\xi}^{0}\right), i=1, \ldots, M \\
\boldsymbol{u}_{, i j} & =-\boldsymbol{K}\left(\boldsymbol{\xi}^{0}\right)^{-1}\left(\boldsymbol{K}_{, i} \boldsymbol{u}_{, j}+\boldsymbol{K}_{, j} \boldsymbol{u}_{, i}+\boldsymbol{K}_{, i j} \boldsymbol{u}\left(\boldsymbol{\xi}^{0}\right)\right), i, j=1, \ldots, M
\end{aligned}
$$


Let $\boldsymbol{u}^{L}(\boldsymbol{\xi})$ and $\boldsymbol{u}^{Q}(\boldsymbol{\xi})$ be the first and second order Taylor approximations of the displacement vector $\boldsymbol{u}(\boldsymbol{\xi})$, respectively. It can be shown straightforwardly that the second order statistics of $\boldsymbol{u}^{L}(\boldsymbol{\xi})$ and $\boldsymbol{u}^{Q}(\boldsymbol{\xi})$ are the following [9,11].

$$
\begin{aligned}
& \mathrm{E}\left[u_{n}^{L}\right]=u_{n}\left(\boldsymbol{\xi}^{0}\right), n=1, \ldots, N_{d} \\
& \operatorname{Cov}\left[u_{n}^{L}, u_{m}^{L}\right]=\sum_{i=1}^{M} \sum_{j=1}^{M} u_{n, i} u_{m, j} \delta_{i j}, n, m=1, \ldots, N_{d} \\
& \mathrm{E}\left[u_{n}^{Q}\right]=u_{n}\left(\boldsymbol{\xi}^{0}\right)+\frac{1}{2} \sum_{i=1}^{M} \sum_{j=1}^{M} u_{n, i j} \delta_{i j}, n=1, \ldots, N_{d} \\
& \operatorname{Cov}\left[u_{n}^{Q}, u_{m}^{Q}\right]=\sum_{i=1}^{M} \sum_{j=1}^{M} u_{n, i} u_{m, j} \delta_{i j}+\frac{1}{4} \sum_{i=1}^{M} \sum_{j=1}^{M} \sum_{k=1}^{M} \sum_{l=1}^{M} u_{n, i j} u_{m, k l}\left(\delta_{i k} \delta_{j l}+\delta_{i l} \delta_{j k}\right), \\
& n, m=1, \ldots, N_{d}
\end{aligned}
$$

In the above equations, $u_{n}^{L}$ is the $n$-th component of $\boldsymbol{u}^{L}(\boldsymbol{\xi}), u_{n}^{Q}$ is the $n$-th component of $\boldsymbol{u}^{Q}(\boldsymbol{\xi}), u_{n}\left(\boldsymbol{\xi}^{0}\right)$ is the $n$-th component of $\boldsymbol{u}\left(\boldsymbol{\xi}^{0}\right), u_{n, i}$ is the $n$-th component of $\boldsymbol{u}_{, i}, u_{n, i j}$ is the $n$-th component of $\boldsymbol{u}_{, i j}$ and $\delta_{i j}$ is the Kronecker delta, which is equal to 1 in case $i=j$ and zero otherwise.

\section{APPROXIMATE REPRESENTATION OF STRUCTURAL RESPONSE CONSID- ERING RECIPROCAL INTERVENING VARIABLES}

In order to overcome the lack of accuracy of first order Taylor expansions, the so-called intervening variables have been applied customarily within the field of structural optimization [5]. This strategy is employed because the response that is being approximated behaves more linearly with respect to these intervening variables. Assuming the intervening variables are defined such that $y_{i}=y_{i}\left(\xi_{i}\right), i=1, \ldots, M$, the expression for approximating the displacement vector $\boldsymbol{u}(\boldsymbol{\xi})$ by means of a first order Taylor expansion with respect to the intervening variables (which is denoted as $\boldsymbol{u}^{I}(\boldsymbol{\xi})$ ) is the following.

$$
\boldsymbol{u}(\boldsymbol{\xi}) \approx \boldsymbol{u}^{I}(\boldsymbol{y}(\boldsymbol{\xi}))=\boldsymbol{u}\left(\boldsymbol{y}\left(\boldsymbol{\xi}^{0}\right)\right)+\left.\sum_{i=1}^{M} \frac{\partial \boldsymbol{u}}{\partial y_{i}}\right|_{\boldsymbol{y}=\boldsymbol{y}\left(\boldsymbol{\xi}^{0}\right)}\left(y_{i}\left(\xi_{i}\right)-y_{i}\left(\xi_{i}^{0}\right)\right)
$$

One of the most commonly used intervening variables is the reciprocal one. Its use is motivated by the fact that for the optimal design of statically determinate trusses where the design variables are the cross section areas of the bars, the displacement vector can be represented exactly by a first order Taylor expansion in case the intermediate variable is selected as reciprocal $[10,6]$. Based on this fact and recalling the objective of this contribution is studying linear static problems where the Young's modulus is characterized as a log-normal random field, the following intervening variable is proposed for constructing a first order Taylor expansion.

$$
y_{i}\left(\xi_{i}\right)=e^{-\alpha_{i} \xi_{i}}, i=1, \ldots, M
$$

In eq. (17), the real, constant coefficient $\alpha_{i}$ is defined such that:

$$
\alpha_{i}=\frac{\sum_{p=1}^{N_{e}}\left|\zeta_{p i}\right|}{\sum_{p=1}^{N_{e}} I\left[\left|\zeta_{p i}\right|>0\right]}
$$


where $|\cdot|$ denotes absolute value and $I[\cdot]$ is an indicator function that is equal to 1 in case its argument holds and zero otherwise.

Considering the reciprocal intervening variable introduced in eq. (17), the first order Taylor expansion of the displacement vector (denoted as $\boldsymbol{u}^{R}(\boldsymbol{\xi})$ ) is the following.

$$
\boldsymbol{u}^{R}(\boldsymbol{\xi})=\boldsymbol{u}\left(\boldsymbol{\xi}^{0}\right)+\sum_{i=1}^{M} \boldsymbol{u}_{, i}\left(\frac{1-e^{-\alpha_{i} \xi_{i}}}{\alpha_{i}}\right)
$$

The second order statistics of $\boldsymbol{u}^{R}(\boldsymbol{\xi})$ are given by the expressions shown below.

$$
\begin{aligned}
\mathrm{E}\left[u_{n}^{R}\right] & =u_{n}\left(\boldsymbol{\xi}^{0}\right)+\sum_{i=1}^{M} u_{n, i}\left(\frac{1-e^{\alpha_{i}^{2} / 2}}{\alpha_{i}}\right), n=1, \ldots, N_{d} \\
\operatorname{Cov}\left[u_{n}^{R}, u_{m}^{R}\right] & =\sum_{i=1}^{M} u_{n, i} u_{m, j}\left(\frac{e^{2 \alpha_{i}^{2}}-e^{\alpha_{i}^{2}}}{\alpha_{i}^{2}}\right), n, m=1, \ldots, N_{d}
\end{aligned}
$$

In the above equations, $u_{n}^{R}$ is the $n$-th component of $\boldsymbol{u}^{R}(\boldsymbol{\xi})$.

\section{NUMERICAL EXAMPLE}

\subsection{Description}

A plate in tension (plain stress) whose Young's modulus is modeled as a log-normal random field is studied. This example has been considered a number of times in the literature, see e.g. [4]. The model is represented schematically in fig. (1). The objective is estimating the second order statistics of the vertical displacement of node in corner D.

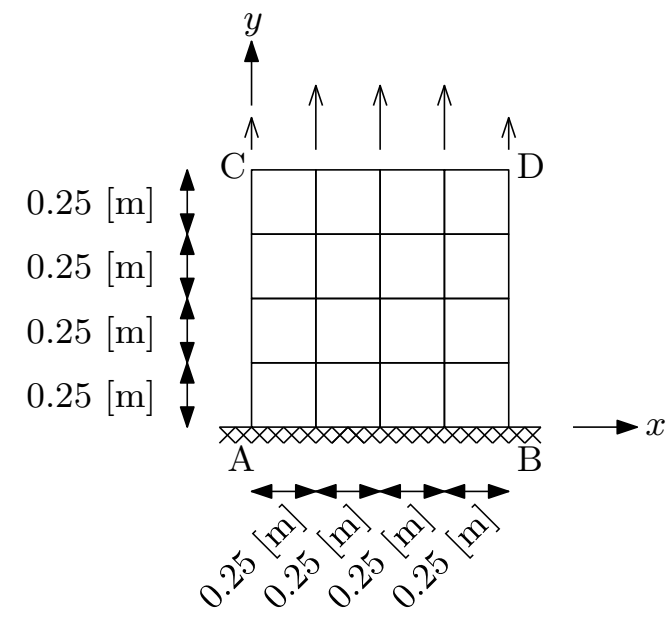

Figure 1. Schematic representation of plate in tension

The height and width of the plate are indicated in fig. (1) while its thickness is 0.01 [m]. The loading consists of a $1[\mathrm{MN}]$ force applied uniformly over the edge CD. The plate is modeled by means of the FEM considering quadrilateral elements of 8 DOF's of dimension $0.25[\mathrm{~m}] \times 0.25[\mathrm{~m}]$. The model comprises a total of $N_{d}=40$ DOF's and $N_{e}=16$ elements. The Young's modulus of the plate is modeled as an homogeneous log-normal discrete random 
field which is discretized using the midpoint method. For representing the underlying Gaussian random field, 16 terms are considered in the K-L expansion. The expected value of the Young's modulus is $200[\mathrm{GPa}]$ and the covariance matrix $\boldsymbol{C}^{E E}$ is:

$$
C_{p q}^{E E}=\sigma^{2} e^{-\frac{\left|\bar{x}_{p}-\bar{x}_{q}\right|}{L}-\frac{\left|\bar{y}_{p}-\bar{y}_{q}\right|}{L}}, p, q=1, \ldots, 16
$$

where $\left(\bar{x}_{p}, \bar{y}_{p}\right)$ are the midpoint coordinates of finite element $p, \sigma^{2}$ is the standard deviation of the Young's modulus and $L=1[\mathrm{~m}]$ is the correlation length. The values assumed by $\sigma^{2}$ vary such that the coefficient of variation of the Young's modulus $\left(\mathrm{CoV}_{\mathrm{E}}\right)$ is within the range $[0.05,0.40]$.

\subsection{Results}

The second order statistics are calculated considering first and second order Taylor expansions involving direct variables (see Section 3) as well as the first order Taylor expansion involving reciprocal intervening variables (see Section 4). The results calculated with these three techniques are compared against the results obtained using Monte Carlo Simulation (MCS) with a total of $1 \times 10^{6}$ samples. The latter results are considered to be the reference. Thus, the accuracy of the different Taylor series expansions is compared in terms of their relative error with respect to MCS. The results obtained are shown in fig. (2) for the error in the estimation of expected value and in fig. (3) for the error in the estimation of the variance. In these figures, the results based on the first order Taylor expansion are denoted as Linear, the second order Taylor expansion as Quadratic and the first order Taylor expansion considering reciprocal intervening variables as Reciprocal.

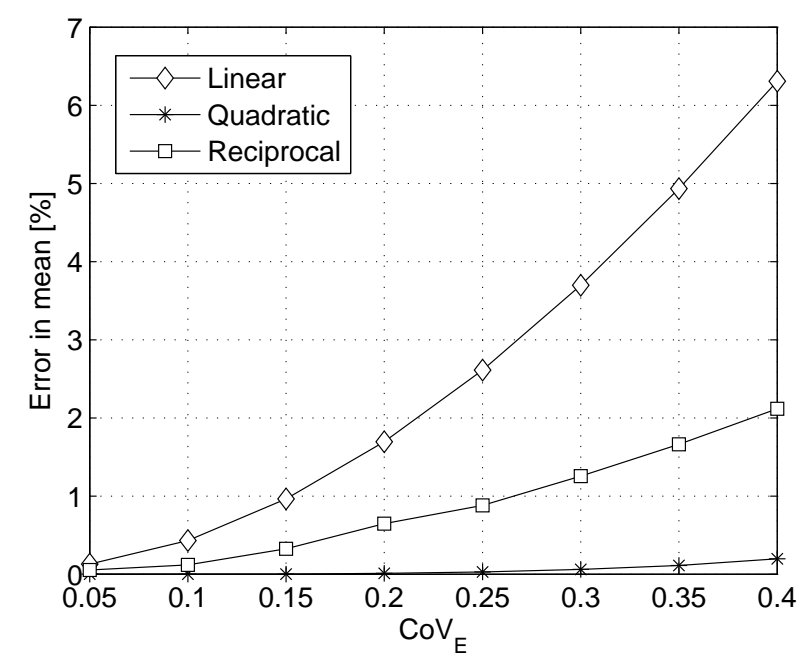

Figure 2. Error in estimation of expected value as a function of the coefficient of variation of the Young's modulus $\left(\mathrm{CoV}_{\mathrm{E}}\right)$

According to the results obtained in figs. (2) and (3), it is observed that the error associated with the estimation of second order statistics is similar for small values of the coefficient of variation of the Young's modulus (below 10\%). However, this situation changes drastically 


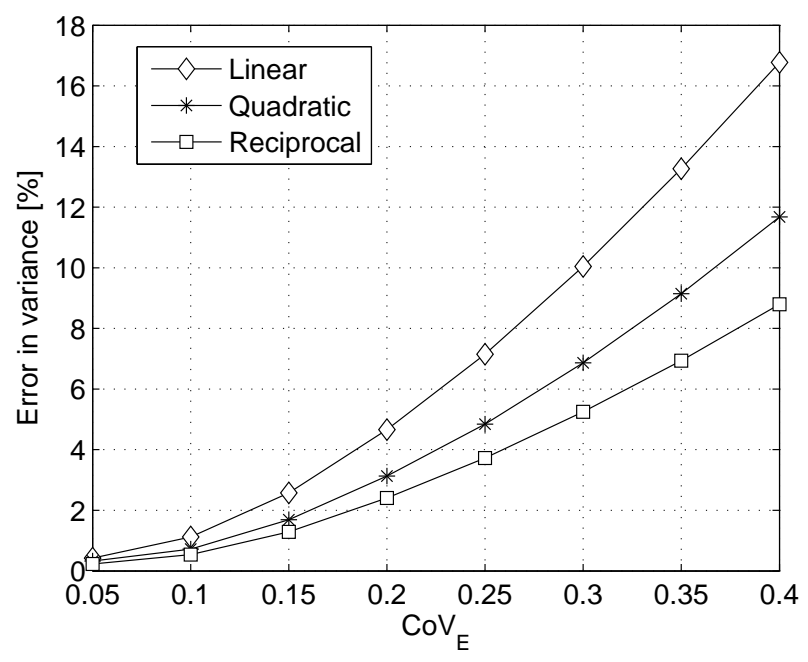

Figure 3. Error in estimation of variance as a function of the coefficient of variation of the Young's modulus $\left(\mathrm{CoV}_{\mathrm{E}}\right)$

as the coefficient of variation increases. For the particular case of fig. (2), it is seen that the most accurate estimate of the mean value is produced by the second order Taylor expansion. However, numerical costs associated with this approach largely exceed the numerical costs associated with the first order Taylor expansion considering either direct or reciprocal variables. For estimating the variance, the first order expansion based on reciprocal variables is the most accurate approach. It should be noted that the numerical costs associated with this expansion are the same as those associated with the first order expansion considering direct variables.

\section{CONCLUSIONS}

In this contribution, a strategy commonly used within the context of structural optimization has been applied for performing stochastic finite element analysis. According to the results obtained, the expansion based on reciprocal intervening variables outperforms expansions based on direct variables. More important, the expansion considering reciprocal variables can be constructed using the very same information required for constructing a first order Taylor series expansion involving direct variables.

Although the results reported herein are promising, it should be kept in mind that the example is of a limited scope. Therefore, future research efforts aim at exploring the application of reciprocal intervening variables to different types of examples involving more challenging structures, comprising a larger number of DOF's and random variables.

\section{Acknowledgements}

This research is partially supported by CONICYT (National Commission for Scientific and Technological Research) under grant number 1110061. This support is gratefully acknowledged by the authors. The authors also wish to thank Professor G.I. Schuëller (University of 
Innsbruck, Austria, EU) for the fruitful discussions while preparing this manuscript.

\section{REFERENCES}

[1] Bathe, K. Finite Element Procedures. Prentice Hall, New Jersey, 1996.

[2] Der Kiureghian, A. and Ke, J.-B. "The stochastic finite element method in structural reliability". Prob. Eng. Mech., 3(2), 83-91, 1988.

[3] Fuchs, M. and Shabtay, E. "The reciprocal approximation in stochastic analysis of structures". Chaos, Solit. \& Fract., 11(6), 889-900, 2000.

[4] Ghanem, R. and Spanos, P. "Polynomial chaos in stochastic finite elements". J. Appl. Mech. (ASME), 57(1), 197-202, 1990.

[5] Haftka, R. and Gürdal, Z. Elements of Structural Optimization. Kluwer, Dordrecht, The Netherlands, $3^{\text {rd }}$ edition, 1992.

[6] Jensen, H.A. and Sepulveda, A.E. "Design sensitivity metric for structural dynamic response" AIAA J., 36(9), 1686-1693, 1998.

[7] Liu, W., Mani, A., and Belytschko, T. "Finite element methods in probabilistic mechanics”. Prob. Eng. Mech., 2(4), 201-213, 1987.

[8] Loève, M. Probability theory. D. Van Nostrand Company, Inc., Princeton, New Jersey, third edition, 1963.

[9] Matthies, H., Brenner, C., Bucher, C., and Soares, C. "Uncertainties in probabilistic numerical analysis of structures and solids - Stochastic finite elements". Struct. Saf., 19(3), 283-336, 1997.

[10] Schmit, L. and Farshi, B. "Some approximation concepts for structural synthesis". AIAA J., 12(5), 692-699, 1974.

[11] Schuëller, G. I. "A state-of-the-art report on computational stochastic mechanics". Prob. Eng. Mech., 12(4), 197-321, 1997.

[12] Stefanou, G. "The stochastic finite element method: Past, present and future". Comp. Meth. Appl. Mech. Eng., 198(9-12), 1031-1051, 2009.

[13] Sudret, B. Stochastic finite element methods and reliability: A state-of-the-art report. Technical report, University of California, Berkeley, 2000. 